\title{
Millimeter Wave Radar for Intelligent Transportation Systems: a Case Study of Multi- Target Problem Solution
}

\author{
Vladimir Kutsov ${ }^{1, *}$, Vladimir Badenko ${ }^{1}$, Sergey Ivanov ${ }^{1}$, and Alexander Fedotov ${ }^{1}$ \\ ${ }^{1}$ Peter the Great St. Petersburg Polytechnic University, 195251, 29 Polytechnic st., St. Petersburg, \\ Russian Federation
}

\begin{abstract}
The influence of millimeter-wave radar receiver noise on the probability of unambiguous determination of unmanned vehicles speed and range in the intelligent transportation system of the "smart city» is investigated. For the proposed new multi-target detection method for FMCW radar, the effect of the technical parameters of the vehicle radars on the required signal-to-noise ratio $(S N R)$ of the receiver is estimated to ensure the probability of true determination of target parameters at $98 \%$.
\end{abstract}

\section{Introduction}

Usually when problems of unmanned vehicle are discussed the two type of vehicles are mentioned: aerial and ground. The applications of Unmanned Aerial Vehicles (UAVs) are attracting increasing attention in recent years because of rapidly growing usage in a wide range of industrial sphere, including regional planning and mapping [1], forestry [2], environmental monitoring [3], logistics [4], agriculture [5] and many other civil application $[6,7]$. Autonomous ground vehicle technology is making a prominent appearance around the world in many forms and related research, testing, and deployment of autonomous vehicles is escalating in many parts of economy [8-10]. One of the main problems remains the determination of the speed, position and other parameters of such moving objects [1114]. For solving such problems, sensor systems based on radar technology are currently widely used $[15,16]$. In this regard, the urgent task is to detect and filter false targets when using millimeter-wave radars [17-20]. Millimeter-wave radar systems for automotive application include pulse radar, frequency- modulated continuous-wave (FMCW) radar and spread spectrum radar.

The main problem of millimeter-wave radar usage for intelligent transportation systems (ITS) are related to signal depolarization, significant attenuation, changes in phase and amplitude during a propagation of such waves, and atmospheric attenuation tends to be higher if the frequency increases, and this also depends on weather conditions [21]. Choosing of a specific frequency band depends on a number of factors such as environment, the backscatter properties of the targets and many others [22]. The basic tasks for millimeter-wave radar usage in ITS are following [23]: detection: decision about the

* Corresponding author: vdkuptsov@yandex.ru 
presence or absence of some targets; target tracking: tracking of the specific target; measurement: a process to estimate the coordinates and parameters the target/targets; recognition: the target belongs or not to the specific class of targets; resolution: detection and measurement of the specific target. Typical key issues that need to be solved for a successful implementation are: cost, number of sensors, problems with choosing the right frequency, installation parameters, sensor fusion, low false positives / targets, etc. [20-24].

There are many methods for solve mentioned tasks. For example, for measurement and position localization several common methods $[25,26]$ such as time difference of arrival (TDOA), differential Doppler (DD) and triangulation are used. An essential challenge that remains open and need to solve is the object detection and classification problem. Radar sensors are a reliable choice for this task, but radar sensors classification capabilities are not optimal, for example, small objects can be easy to confuse with birds, and in most cases they are difficult to distinguish [27]. The most commonly used characteristic of a radar signal for automatic target classification is the micro-Doppler signature [28]. A surveillance radar is operating with a rotating antenna to detect and track multiple target [29]. Deep learning based methodologies has some success for tasks concerning radar data. But such approaches require a large amount of ground truth annotated data and can only be performed by an expert in the field. Also acquired raw data from a radar sensor may not be enough and sophisticated radar signal processing is required to extract spatiotemporal and intrinsic information for a deep learning process $[28,30]$.

Freight and logistics of transport in the «smart city» determines the need to determine the position of vehicles and UAVs and predict their course of movement. A distinctive feature of the real situation of unmanned vehicles (UV) and UAV movement is the simultaneous presence of a significant number of vehicles in the «smart city», which determines the need to distinguish vehicles in radars by location and speed. To solve this problem, in [14] we proposed a method for estimating speeds and distances to targets in multi-target mode, which allowed us to significantly expand the range of uniquely determined vehicle speeds.

The objective of the paper is a study of the noise properties of the proposed multi-target detection method for FMCW radar [14], namely, the influence of the technical parameters of vehicle's radars in intelligent transport systems on the required signal-to-noise ratio $(S N R)$ of the receiver and providing the probability of true determination of target parameters at the level of $98 \%$.

\section{Multi-target Detection Method for FMCW Radar}

To solve the issue of determining the location and speed of vehicles, various forms of radio signals are used. Based on the form of the radio signal shown in figure 1, which is quite effective for realizing in the radar, we proposed a method for eliminating incorrect correspondences of the locations and speeds of targets in the multiple target mode [14].

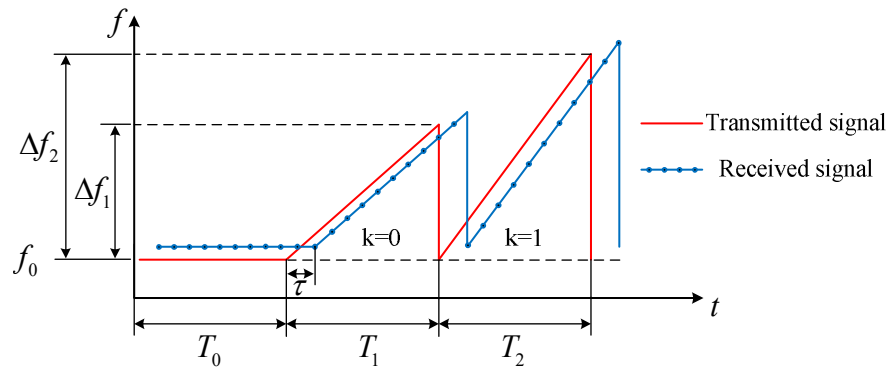

Fig. 1. Proposed Waveform [14]. 
In the mixer of the vehicle's radar receiver, an intermediate frequency $(I F)$ signal is generated equal to the frequency difference between the transmitter and receiver at each time point. The intermediate frequency, called the beat frequency, is digitized by the analog-to-digital converter (ADC) with the sampling frequency of the $f_{S}$. The beat frequencies $f_{R 1}$ and $f_{R 2}$, i.e. the frequency of the signal at the output of the mixer in the quadrature channels $I$ and $Q$ of the intermediate frequency of the radar receiver for chirps with numbers $k=0$ and $k=1$, respectively, are determined by the expressions

$$
\begin{aligned}
& f_{R 1}=\frac{\Delta f_{1}}{T_{1}} \tau_{0}-\frac{2 V_{R}}{c} f_{0}, \\
& f_{R 2}=\frac{\Delta f_{2}}{T_{2}} \tau_{0}-\frac{2 V_{R}}{c}\left(f_{0}-\frac{3 \Delta f_{2}}{2}\right)
\end{aligned}
$$

The problem of determining the parameters of targets in multi-target mode is that for each speed from the set of speeds of all targets, it is necessary to match the true range to the target from the set of all ranges to the targets. Based on the refined model of the radar signal IF [18] we found [14] that the minimum of functional $\Xi\left(f_{R 1}, f_{R 2}\right)$ correspond to a true pair of speed-distance of targets

$$
\Xi\left(f_{R 1}, f_{R 2}\right)=f_{R 1} \frac{T_{1}}{\Delta f_{1}}-f_{R 2} \frac{T_{2}}{\Delta f_{2}}-f_{D}\left(\frac{T_{1}}{\Delta f_{1}}-\frac{T_{2}}{\Delta f_{2}}\right) \rightarrow \min
$$

The implementation of the method in vehicle radars consists of a sequence of calculations: 1. one-dimensional fast complex Fourier transform of the samples of the intermediate frequency signal, 2. estimation of the frequencies of the maxima of the obtained amplitude spectrum, 3. calculation of the ranges and speeds of the targets by estimating the frequencies of the maximums of the amplitude spectrum, 4. search of the minimum functional (3) for various combinations of pairs of Doppler frequency and beat frequencies corresponding to the range $\mathrm{R}$ to the target. The Doppler frequency determines the speed of the vehicle in accordance with the expression $f_{D}=2 \cdot V \cdot f_{0} / c$, where $f_{0}$ is the radio frequency of the radar, and $c$ is the speed of light.

Of practical interest for vehicle radar developers is the conscious choice of the technical parameters of the radar, which ensures its high-quality operation at reasonable production costs. As a characteristic of the quality of work, it is possible to use the probability of a true determination of the $\mathrm{P}_{\text {trueVR }}$ parameters. The cost of manufacturing a radar vehicle is reduced, including when using the elemental base of electronic components that perform their functions not at the currently limiting signal processing frequencies.

\section{Result and discussion}

The shape of the radar signal, its radiated power and radio frequency, as well as the range and propagation conditions of the radio waves to the vehicle, determine the signal-to-noise ratio in the radar radio receiver. It is the signal-to-noise ratio that determines the quality of the radar, which can be estimated as the probability of a true determination of the target parameters.

Recommendations on the selection of technical parameters for vehicle radars are based on modeling the operation of a vehicle radar in multi-target mode in the LabVIEW programming environment. The carrier frequency $f_{0}$ was taken equal to $76,5 \mathrm{GHz}$, sweep bandwidth $\Delta f_{1}=450$ and $\Delta f_{2}=1000 \mathrm{MHz}$ respectively. The technical parameters of the radar - the duration of the waveform segments, the sampling frequency of the ADC and the number of simultaneously recognized targets varied during the computational experiments. 
The signal-to-noise ratio was determined in accordance with the expression

$$
S N R=10 \cdot \lg \frac{P_{b e a t}}{2 \sigma^{2}},
$$

where $\mathrm{P}_{\text {beat }}$ - beat signal power, $\sigma^{2}$ - Gaussian noise variance. Noise was determined by integration over the entire frequency band of the fast Fourier transform (FFT).

For a given signal-to-noise ratio and waveform parameters of the radio signal, 500 computer experiments were conducted to determine the true values of the speed and range of many targets. If the parameters of at least one of the targets were determined incorrectly in the experiment, the result was recorded as negative. If the parameters of all $N$ goals were determined without errors, the result was recorded as positive. The criterion for distinguishing between positive and negative experimental results was the condition that the standard deviation of the velocity value for five hundred experiments did not exceed 1 $\mathrm{km} / \mathrm{h}$ and the standard deviation of the range did not exceed $1 \mathrm{~m}$. Probability of target velocity and distance true estimation was calculated as the ratio of the number of experiments with positive results to the total number of experiments equal to 500 .

A strong dependence of the probability of the true determination of the target parameters on the sampling frequency, the duration of the waveform segments and the number of simultaneously determined targets is revealed. Figure 2 shows the dependence of the probability of the true determination of the parameters on the $S N R$ at various sampling frequencies of the ADC. With an increase in the sampling rate, to achieve a high probability of true determination of the target parameters (at the level of $P_{\text {trueVR }}=98 \%$ ), a significantly lower corresponding $S N R_{98 \%}$ value is required. The probability of the unambiguous determination of parameters is especially degraded when the sampling frequency is reduced from $500 \mathrm{kHz}$ to $250 \mathrm{kHz}$. With such a change in the sampling frequency, to achieve $P_{\text {trueVR }}=98 \%$, an increase in $S N R$ of $7 \mathrm{~dB}$ is required, which inevitably leads to the need to increase the power of the radar transmitter, or to reduce the maximum detectable range to the target. Tuning the radar to a sampling frequency above $1 \mathrm{MHz}$ results in a not so strong reduction in the required $S N R$. So when you increase the sampling frequency from 1 to 5 $\mathrm{MHz}$, the $S N R$ will drop by only $2 \mathrm{~dB}$. But an increase in the sampling frequency entails an increase in the cost of vehicle radars and an increase in energy consumption, which is very important for stand-alone battery radars. Thus, there is a certain optimum in the choice of the sampling frequency of the vehicle radar, which also depends on the maximum range determined by the radar to the target. In our computational experiments, for the chosen simulation parameters, the optimal sampling frequency of the ADC of the vehicle radar was in the vicinity of $f_{S}=1 \mathrm{MHz}$.

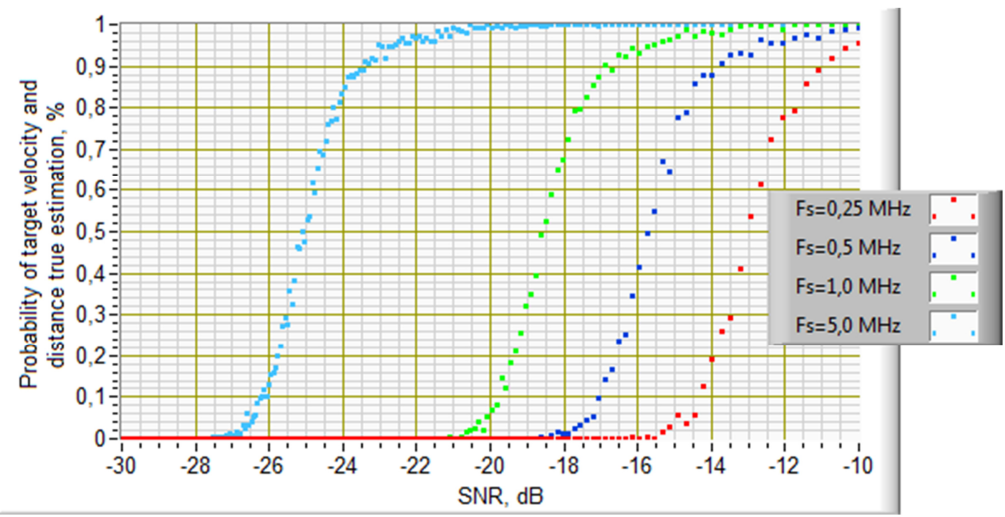

Fig. 2. The dependency of probability of unambiguous determination of the $P_{\text {trueVR }}$ from $S N R$ for various ADC sampling frequencies $(T=8.192 \mathrm{~ms}, N=4)$. 
The dependency of probability of unambiguous determination of the $P_{\text {trueVR }}$ from $S N R$ at various lengths of radar waveform segments of vehicle radar $\left(T_{0}=T_{1}=T_{2}=T\right.$ on figure 1$)$ is shown on figure 3 .

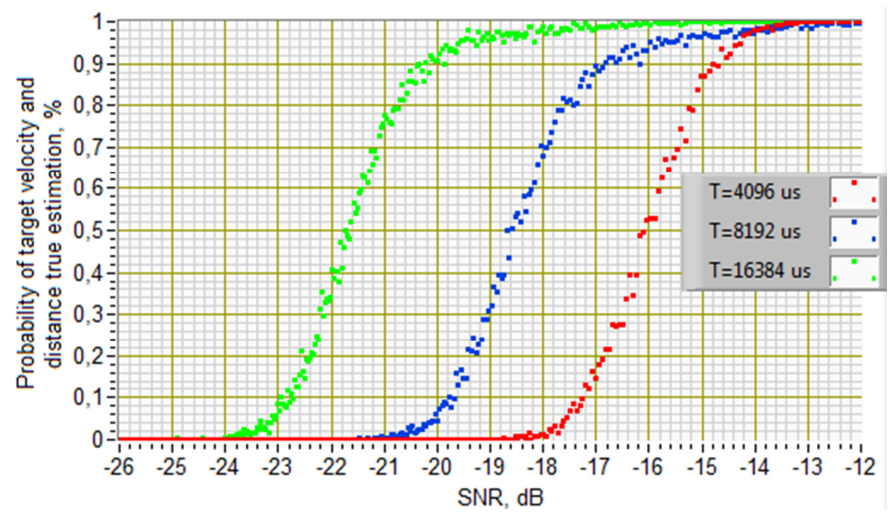

Fig. 3. Probability of unambiguous determination of the $P_{\text {trueVR }}$ from $S N R$ at various segment lengths $\left(f_{S}=1 \mathrm{MHz}, N=4\right)$

A doubling of the duration of the segments leads to a decrease in the required $S N R_{98 \%}$ by $2-3 \mathrm{~dB}$. However, it is not possible to significantly increase the duration of the segments, since in this case, at high target speeds, the target moves a significant distance during the measurement. So, for example, at a target speed of $100 \mathrm{~km} / \mathrm{h}$ during a measurement time of $50 \mathrm{~ms}$ (three segments of $16.384 \mathrm{~ms}$ ), the target will travel a distance of $1.4 \mathrm{~m}$, which can be unacceptably crude accuracy. Due to this reason, the reasonable duration of the segments does not exceed $16.384 \mathrm{~ms}$. Such an exact value of the segment duration is determined by the number of samples of the Fourier transform multiple of degree 2. In this case, a fast Fourier transform is used, which significantly reduces the conversion time compared to a simple discrete Fourier transform.

The dependence of the probability of unambiguous determination of parameters $\mathrm{P}_{\text {trueVR }}$ from $S N R$ with various amounts of simultaneously recognized targets $N$ from 2 up to 12 is shown on figure 4.

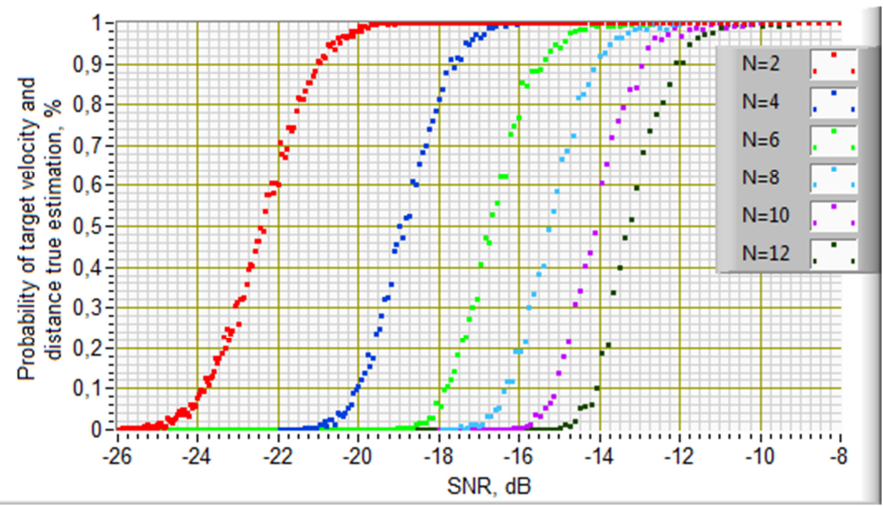

Fig. 4. Dependence of the probability of unambiguous determination of parameters $\mathrm{P}_{\text {trueVR }}$ from $S N R$ with various amounts of simultaneously recognized targets $\left(T=8.192 \mathrm{~ms}, f_{S}=1\right.$ $\mathrm{MHz})$. 
With an increase in the number of simultaneously evaluated targets, the probability of a true determination of the speed and range of targets, which is $98 \%$, is achieved with a larger signal-to-noise ratio. For example, for 2 targets the $98 \%$ probability of target velocity and distance true estimation equals to $S N R_{98 \%}=-20 \mathrm{~dB}$, and for 12 targets $S N R_{98 \%}=-10 \mathrm{~dB}$. Thus, adding each target to the multi-target algorithm makes it necessary to increase the $S N R$ by approximately $\triangle S N R=1-2 \mathrm{~dB}$ for $N$ in the first ten and more than $4 d B$ for $N$ in the second ten. As the number of targets increases, the $\triangle S N R$ value increases.

The decrease of probability of target velocity and distance unambiguous estimation near $S N R_{98 \%}$ values happen dramatically, when changing $S N R$ by $4 \mathrm{~dB}$ probability of target velocity and distance unambiguous estimation falls from one to zero. Identified dependencies for probability of target velocity and distance unambiguous estimation determine the need for a significant reserve in $S N R$ when designing radars for multi-target mode, that remotely senses the parameters of small unmanned vehicles.

\section{Conclusion}

The determination of the UV and UAV motion parameters in the intelligent transport systems of the «smart city» technology is carried out using millimeter-wave radars. A distinctive feature of the real situation of UV and UAV movement is the presence in the zone of analysis of the transport situation at the same time of a significant number of vehicles, the parameters of which must be determined. This article investigates the dependence of the probability of unambiguous determination of target parameters in the multi-target mode on the signal-to-noise ratio in the radar receiver. Quantitative patterns of the influence of the technical parameters of radars on the probability of an unambiguous determination of target parameters are identified. Recommendations on the selection of technical parameters for vehicle radars are based on modeling the operation of a vehicle radar in multi-target mode in the LabVIEW programming environment and can be used by developers to create radars optimized in terms of price / quality.

The reported study was funded by RFBR, project number 19-29-06034.

\section{References}

1. F. Nex, F. Remondino, Applied geomatics, 6(1), 1-15, (2014). DOI: 10.1007/s12518013-0120-x

2. J. Iglhaut, C. Cabo, S. Puliti, L. Piermattei, J. O’Connor, J. Rosette, Curr. For. Rep., 5(3), 155-168, (2019). DOI: 10.1007/s40725-019-00094-3

3. T. F. Villa, F. Gonzalez, B. Miljievic, Z. D. Ristovski, L. Morawska, Sensors, 16(7), 1072, (2016). DOI: 10.3390/s16071072

4. J. P. Škrinjar, P. Škorput, M. Furdić, Lecture Notes in Networks and Systems, 42, 359366, (2018). DOI: 10.1007/978-3-319-90893-9_43.

5. S. Yang, X. Yang, J. Mo, Precis. Agric. 19(2), 278-292, (2018). DOI: 10.1007/s11119017-9516-7

6. A. Otto, N. Agatz, J. Campbell, B. Golden, E. Pesch, Networks, 72(4), 411-458, (2018). DOI: 10.1002/net.21818

7. H. Yao, R. Qin, X. Chen, Remote Sens-Basel, 11(12), 1443, (2019). DOI:10.3390/rs11121443

8. J. Van Brummelen, M. O’Brien, D. Gruyer, H. Najjaran, Transport. Res. C-Emer. 89, 384-406, (2018). DOI: 10.1016/j.trc.2018.02.012 
9. A. Soni, H. Hu, Robotics, 7(4), 67, (2018). doi:10.3390/robotics7040067

10. R. Cajo, T.T.Mac, D. Plaza, C. Copot, R. De Keyser, C. Ionescu, IEEE Access, 7, 66864-66878, (2019). DOI: 10.1109/ACCESS.2019.2918578

11. J. Yoon, M. Feng, H. Xu, AIAA Scitech 2019 Forum, 2072, (2019). DOI: $10.2514 / 6.2019-2072$

12. A. Stateczny, W. Kazimierski, D. Gronska-Sledz, W. Motyl, Remote Sens-Basel, 11(10), 1156, (2019). DOI: 10.3390/rs11101156

13. R. P. Guan, B. Ristic, L. Wang, B. Moran, R. Evans, Int. J. Control, 90(4), 888-900, (2017). DOI: $10.1080 / 00207179.2016 .1244727$

14. V. D. Kuptsov, S. I. Ivanov, A. A. Fedotov, V. L. Badenko, Lect. Notes Comput. Sc. 11660, 355-364, (2019). DOI: 10.1007/978-3-030-30859-9_30

15. Z. Yang, L. S. Pun-Cheng, Image Vision Comput. 69, 143-154, (2018). DOI: 10.1016/j.imavis.2017.09.008

16. R. Pérez, F. Schubert, R. Rasshofer, E. Biebl, Adv. Radio Sci. 17, 129-136, (2019). DOI:10.5194/ars-17-129-2019

17. W. Wang, J. Du, J. Gao, Sensors, 18(10), 3386, (2018). DOI:10.3390/s18103386

18. S. I. Ivanov, V. D. Kuptsov, A. A Fedotov, J. Phys.: Conf. Ser. 1236(1), 012081, (2019). DOI:10.1088/1742-6596/1236/1/012081

19. J. Steinbaeck, C., Steger, G., Holweg, N. Druml, In 2017 Sensor Data Fusion: Trends, Solutions, Applications, 1-6, (2017). DOI: 10.1109/SDF.2017.8126389

20. S. Honma, N. Uehara, Signal, 1, (2001)

21. Z. Xiao, D. Yang, F. Wen, K. Jiang, Sensors, 19(9), 1967, (2019). DOI:10.3390/s19091967

22. X. Wang, L. Xu, H. Sun, J. Xin, N. Zheng, IEEE Trans. Intell. Transp. Syst. 17(7), 2075-2084, (2016). DOI: 10.1109/TITS.2016.2533542

23. J. Moghaddasi, K. Wu, IEEE Access, 4, 818-838, (2016). DOI: 10.1109/ACCESS.2016.2530979

24. F. Arena, G. Pau, Future Internet, 11(2), 27, (2019). DOI: 10.3390/fi11020027

25. S. Van Doan, J. Vesely, 16th International Radar Symposium, 800-805, (2015). DOI:10.1109/IRS.2015.7226350

26. T. Tirer, A.J. Weiss, Signal Process. 141, 288-298, (2017). DOI: 10.1016/j.sigpro.2017.06.019

27. S. Samaras, E. Diamantidou, D. Ataloglou, N. Sakellariou, A. Vafeiadis, V. Magoulianitis, ..., P. Daras, Sensors, 19(22), 4837, (2019). DOI:10.3390/s19224837

28. Y. Ding, J. Tang, IEEE Trans. Geosci. Remote Sens., 52(9), 5807-5819, (2014). DOI: 10.1109/TGRS.2013.2292826

29. H. Taheri, A.M.I.N.A. El. Gennouni, A. Lyhyaoui, J. Theor. Appl. Inf. Technol. 97(13), 3604-3615, (2019)

30. A. Carrio, C. Sampedro, A. Rodriguez-Ramos, P. Campoy, J. Sensors, 2017, 3296874, (2017). DOI: $10.1155 / 2017 / 3296874$ 